\title{
Electroweak physics at the LHC
}

\section{Lucia DI CIACCIO*†}

On behalf of ATLAS and CMS Collaborations

LAPP, Annecy

E-mail: lucia.di.ciaccio@cern.ch

Precise measurements of electroweak parameters represent an important aspect of the LHC physics program. This paper discusses the measurement of the $W$ boson mass, of the top quark mass, of the $Z$ forward-backward asymmetry and of the production cross-section of boson pairs. The high center of mass energy of LHC and the large statistics samples of $W$, top, $Z$ and diboson events that will be collected by the two general-purpose detectors ATLAS and CMS, will allow to improve the precision already achieved on the discussed parameters.

Physics at LHC 2008

29 September - October 4, 2008

Split, Croatia

\footnotetext{
${ }^{*}$ Speaker.

${ }^{\dagger}$ It is a pleasure for me to thank my colleagues of ATLAS and CMS.
} 


\section{Introduction}

The main parameters of the electroweak theory are measured with very good accuracy [1]. In particular, the mass of the $W$ boson is known nowadays with an uncertainty of $0.03 \%$, the uncertainty on the top quark mass is $0.7 \%$ and the uncertainty on the electroweak-mixing angle is $0.07 \%$. The electroweak fit combines these measurements and indicates that the present theory is very successfull [1]. The studies of the boson pair production, done by the LEP and Tevatron experiments allow to derive limits on the triple gauge boson couplings and do not indicate any deviation from the electroweak predictions.

The main aim of improving further the precision on the above mentioned parameters by measuring them at LHC, is to constrain in a tighter way the Standard Model Higgs mass, and to perform a more stringent consistency test of the theory, hoping that deviations from the expected behaviour will finally show up.

In one year, the ATLAS and CMS experiments [2] expect to collect $30 \mathrm{fb}^{-1}$ of data each, during the "low luminosity" phase of the accelerator and $100 \mathrm{fb}^{-1}$ each, during the "high luminosity" phase. The integrated luminosity collected so far at Tevatron is $\approx 5 \mathrm{fb}^{-1}$.

In the next sections, the measurements of the $W$ boson mass, of the top quark mass, of the $Z$ forward-backward asymmetry and of the production cross-section of boson pairs by the ATLAS and CMS collaborations will be reviewed and the expected accuracies will be discussed.

\section{2. $W$ Mass Measurements}

The $W$ mass, $M_{W}$, has been measured at SppsS, at LEP2 and at Tevatron [3]. At LHC there is a great potential for improvements: the $W$ and $Z$ cross-sections are one order of magnitude higher than at Tevatron $\left(\sigma_{N N L O}^{14 T e V}(p p \rightarrow W(\ell v) X) \approx 20 \mathrm{nb}, \sigma_{N N L O}^{14 T e V}(p p \rightarrow Z(\ell \ell) X) \approx 2 \mathrm{nb}\right)$ and the design peak luminosity $\left(\mathrm{L} \approx 10^{34} \mathrm{~cm}^{-2} \mathrm{~s}^{-1}\right)$ is about ten times higher than the Tevatron design peak luminosity. The signature of events with $W \rightarrow \ell v$ decays is given by an isolated lepton of high transverse momentum $\left(p_{T}^{\ell}\right)$ and missing transverse energy $\left(\mathrm{E}_{T}^{\text {miss }}\right)$ due to the neutrino. Additional selection criteria on the transverse momentum of the hadronic system recoiling against the $W$ boson are used and no event with jets of high transverse momentum is allowed. The total selection efficiency of these cuts is $\varepsilon_{s e l} \approx 20 \%$. For an integrated luminosity of $1 \mathrm{fb}^{-1}, 4$ million events with $W \rightarrow \ell v$ $(\ell=\mathrm{e}$ or $\mu$ ) decays are expected.

The $W$ mass is extracted from the measured $p_{T}^{\ell}$ distribution or from the Jacobian peak observed in the transverse mass of the lepton-neutrino system, $M_{T}^{W}$. The analyses exploiting these two variables are complementary to each other [4]: the main systematic effect on the $p_{T}^{\ell}$ distribution shape is the amount of transverse momentum, $p_{T}^{W}$, carried by the $W$ boson while the main systematic effect on the $M_{T}^{W}$ distribution shape is related to detector resolution effects.

The $W$ mass is obtained by comparing the measured distributions with template distributions generated from data ( $Z$ events are used), thus no longer relying on MC simulations. To build templates, in the CMS analyses [4] two complementary methods have been developed, the first applied to the electron channel, the second to the muon channel. In the scaled observable method, the template distributions are created by transforming $Z$ distributions (e.g. $p_{T}^{\ell}$ or $Z$ transverse mass distribution ) into $W$ ones (see Fig. 1). For $1 \mathrm{fb}^{-1}$ of integrated luminosity, the electron channel gives a 
total uncertainty $\Delta M_{W}=40$ (stat.) \pm 40 (syst.exp.) \pm 40 (syst.theo.) $\mathrm{MeV}$, where the uncertainty on the lepton energy linearity dominates the experimental systematic error and the uncertainty on the $p_{T}^{W}$ dominates the theoretical systematic error. The second method to build templates is based on an event-by-event transformation to change a $Z$ event into a $W$ event corresponding to a trial value of the $M_{W}$. For $1 \mathrm{fb}^{-1}$ of integrated luminosity, the muon channel gives an uncertainty $\Delta M_{W}=40$ (stat.) \pm 64 (syst.exp.) \pm 20 (syst.theo.) $\mathrm{MeV}$, where the uncertainty on the $\mathrm{E}_{T}^{\text {miss }}$ dominates the experimental systematic error, while the uncertainty on the Parton Distribution Functions(PDF) dominates the theoretical systematic error.

In the ATLAS study [5] the template distributions are obtained by convoluting the generated distribution of $p_{T}^{\ell}$ or of $M_{t}^{W}$, with the measured detector response. The detector response is obtained using $Z$ events. With $15 \mathrm{pb}^{-1}$, the electron transverse momentum analysis yields a precision of $\Delta M_{W}=120$ (stat) \pm 117 (syst.) MeV. The systematic uncertainty is dominated by the lepton energy scale. In the muon channel, using $15 \mathrm{pb}^{-1}$, the transverse mass analysis gives, $\Delta M_{W}=57$ (stat) \pm 231 (syst.), where the dominant contribution comes from the recoil calibration. PDF uncertainties contributes $\Delta M_{W}=25 \mathrm{MeV}$. Obviously, due to the limited statistics, the experimental error obtained in the ATLAS analyses is not competitive with the previous CMS results. The interest of the ATLAS results reside in establishing what can be done at the beginning of the data taking by using mainly data driven methods.

Strategies to pin down the theoretical error are described in [6]. Two sources of theoretical error are discussed in the following: the uncertainty related to $p_{T}^{W}$ and to the $W$ rapidity $(y)$. The $p_{T}^{W}$ distribution is mainly the result of the intrinsic $p_{T}$ of the incoming partons and of the initial state radiation. These mechanisms can be constrained with dilepton events. The Drell-Yan continuum, between 20 $\mathrm{GeV}$ and $\mathrm{M}_{Z}$, provides a strong lever arm on the dilepton invariant mass and allows to measure the dilepton $p_{T}$ distribution in the $W$ mass range. The $W$ rapidity distribution is essentially driven by the proton structure functions. At the LHC, $Z$ and $W$ bosons are essentially produced through sea quark interactions; therefore a strong correlation between the $W$ and $Z$ production is expected. In particular, a precise measurement of $d \sigma / d y(Z)$ will constrain the $W$ rapidity distribution. In [6], it has been estimated that using these methods and with $10 \mathrm{fb}^{-1}$, the total systematic error in a single $M_{W}$ analysis could be brought below $\approx 10 \mathrm{MeV}$.

\section{Top Mass Measurements}

The LHC experiments have a great potential to measure precisely the top mass $\left(M_{t}\right)$ : top quark pairs, mainly produced via gluon fusion, yields a production cross-section of $833 \mathrm{pb}$, at next to leading order, $\approx 100$ times higher than at Tevatron. It is expected that the $M_{t}$ measurements at LHC will become soon limited by the systematic error.

Many studies have been performed by ATLAS [5] and CMS [7, 8]. The "golden" channel is the semi-leptonic channel: $t \bar{t} \rightarrow W b+W \bar{b} \rightarrow(\ell v) b+(j j) \bar{b}$, where the topology of the final state can be exploited to select the signal with good purity, and the hadronic side is used to measure the top quark mass. These events are selected by requiring an isolated high $p_{T}$ lepton, $\mathrm{E}_{T}^{\text {miss }}$ and at least 4 jets, two of which $b$-tagged. This gives a signal efficiency of $\approx 5 \%$ with a signal over background ratio of the order of 10 [5]. The main backgrounds are single top events, mainly reduced by the 4 jet cut, fully hadronic $t \bar{t}$ events, reduced by the lepton requirements, $W+$ jet and $Z+$ jet events. 


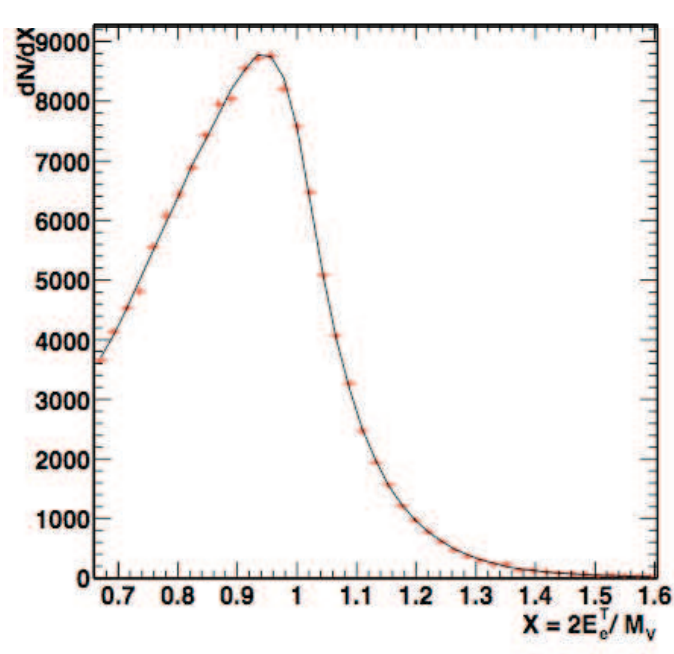

Figure 1: Comparison of the scaled electron spectra for $Z$ (dots) and $W$ boson (line) events in the CMS analysis[4]. $\mathrm{E}_{\mathrm{e}}^{\mathrm{T}}$ is the transverse electron energy and $\mathrm{M}_{\mathrm{V}}$ is the boson mass $(\mathrm{V}=Z$ or $W)$.

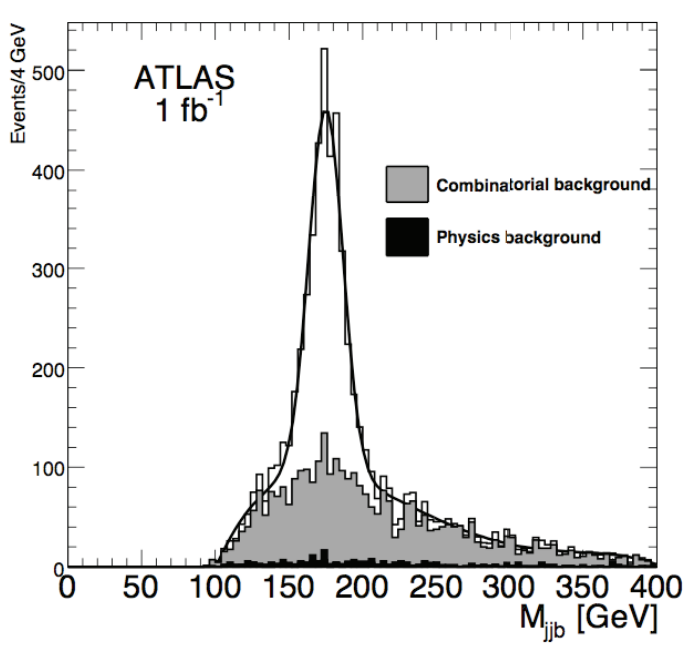

Figure 2: The hadronic top quark mass, $\mathbf{M}_{j j b}$, fitted with the sum of a Gaussian and a third order polynomial [5].

Backgrounds from multi-jet events and $b \bar{b}$ production are negligible after the leptonic selections and backgrounds from diboson events have a much smaller contribution beeing strongly reduced by cuts on jets. To reconstruct the hadronic side of the decay, an in situ rescaling is performed by a minimization procedure. The minimization constrains the light jet pair mass to $M_{W}$, via corrections to the light jet energies. All possible jet combinations are tried; the one minimizing the $X^{2}$ is kept. The $b$-jet closest to the hadronic $W$ boson is associated to the chosen pair. The three jet invariant mass is then fitted with a Gaussian plus a polynomial (see Fig.2). The result is $M_{t}=175.0 \pm 0.2$ (stat.) \pm 1.0 (syst.) $\mathrm{GeV}$, for an input mass of $175 \mathrm{GeV}$ and $1 \mathrm{fb}^{-1}$. The precision on $M_{t}$ relies mainly on the control of the $b$-jet energy scale uncertainty: a precision of the order of 1 to $3.5 \mathrm{GeV}$ should be achievable with $1 \mathrm{fb}^{-1}$, and a $b$-jet energy scale uncertainty of 1 to $5 \%$.

Events with one or no $b$-tagged jets lead also to an interesting measurement if the background shape is constrained from data (using side-band or event mixing techniques, as explained in [5]). For 1 $\mathrm{fb}^{-1}$ the estimated precision on $M_{t}$ in the ATLAS study [5] which doesn't use b-tag, is below 2 $\mathrm{GeV}$ (assuming a jet energy scale uncertainty of the order of the percent). These samples are thus useful for jet energy scale or b-tagging studies during the commissioning phase with early data.

Another possibility for determining $M_{t}$ exploits final states with $J / \Psi$ from the fragmentation of the $\mathrm{b}$ quark [9]. The top quark mass is determined via its correlation with the invariant mass of the $J / \Psi(\rightarrow \ell \ell)$ and the lepton from the $W$ decay coming from the same top. A study of CMS [10] finds that in $100 \mathrm{fb}^{-1}$ of data, $\sim 1000$ events are selected. It is particularly relevant that this analysis reduces to a minimum those systematics which are expected to dominate in more traditional estimations of $M_{t}$. A total error $\Delta M_{t} \sim 2 \mathrm{GeV}$, dominated by theoretical uncertainties (scale definition and $b$-fragmentation effects) is well within reach. It is expected that a better understanding of the 
theoretical systematics at the time when the measurement will be made is possible and will bring $\Delta M_{t} \sim 1 \mathrm{GeV}$.

A last possibility is to estimate $M_{t}$ from the measured $t \bar{t}$ production cross section [11]. This would allow a determination independent of the kinematic reconstruction. However, even without considering experimental uncertainties, the achievable precision would already be limited to $2 \mathrm{GeV}$ due to uncertainties on the theoretical calculations. These uncertainties can be reduced by performing the computation at higher orders, including the resummation of next-to-leading logarithmic corrections[12].

\section{Forward-Backward Asymmetry in $Z$ Decays}

The measurement of the forward-backward asymmetry $\left(\mathrm{A}_{F B}\right)$ of the $\gamma^{*} / Z$ decay products probes the V-A structure of the electroweak theory and can be used to extract indirectly the effective weak mixing angle, $\sin ^{2} \theta_{e f f}$. This measurement is also interesting since the exchange of a new particle $\mathrm{X}$ in the process $f \bar{f} \rightarrow X \rightarrow \ell \bar{\ell}$, alters the value of $\mathrm{A}_{F B}$.

LEP and SLD have measured very precisely $\sin ^{2} \theta_{e f f}$. The world average value is $\sin ^{2} \theta_{\text {eff }}=$ $0.23153 \pm 0.00016$ [1]. More recently, using $1.1 \mathrm{fb}^{-1}$ of data, the D0 experiment at $p \bar{p}$ Tevatron collider, has measured $\mathrm{A}_{F B}$ in $Z$ decays and extracted $\sin ^{2} \theta_{\text {eff }}$ with a statistical error of 0.0018 and a systematic error (by far dominated by PDF related uncertainties) of 0.0006 [1].

Even in $p p$ collisions, a forward-backward asymmetry of the $Z$ decay products is expected. The $Z$ is formed by a quark-antiquark pair; while the anti-quark always arises from the sea, the quark may also be a valence quark which on average carries a higher momentum than sea quarks. Thus the boost direction of the $\mathrm{Z}$ indicates the quark direction. This assumption is better verified when the $Z$ decay product (i.e. the dilepton pair) has high rapidity. In order to improve the measurement precision, it will be therefore necessary to detect leptons in the very forward rapidity regions, and this favors the electron over the muon $Z$ decay channel. In ATLAS the forward calorimeter FCAL extends the electron indentification capability up to $\eta=4.9$ (the muon spectrometer covers the region up to $\eta=2.7)$. In the ATLAS $\mathrm{A}_{F B}$ study [5] it is required that one of the electrons lies within $|\eta|<2.5$, while the other can go up to $|\eta|=4.9$. In the region $2.5<|\eta|<4$.9 an electron identification efficiency of $80 \%$ is achieved with less than 3\% QCD background. The statistical uncertainty is $\Delta \mathrm{A}_{F B}=2.7 * 10^{-4}$.

The measured asymmetry can be interpreted as a measurement of $\sin ^{2} \theta_{\text {eff }}$. With $100 \mathrm{fb}^{-1}$ of luminosity, ATLAS [5] expects a statistical uncertainty on $\sin ^{2} \theta_{\text {eff }}$ of 0.00015 and a systematic uncertainty of 0.00024 (by far dominated by PDF related uncertainties), comparable to the uncertainty of the present world average. It is expect that in the future the knowledge of the PDFs will improve thanks to the constraints imposed by Tevatron, HERA and LHC measurements (e.g. using $W$ asymmetry). By the time the high luminosity data will be available the systematic uncertainty on $\sin ^{2} \theta_{\text {eff }}$ should therefore decrease. If this is not the case, the $\mathrm{A}_{F B}$ measurement can be used, conversely, to constrain the PDFs.

\section{Associated Production of Gauge Bosons}

The study of the pair-production of electroweak gauge bosons tests the triple gauge boson cou- 


\begin{tabular}{|c|ccccc|}
\hline \hline Dibosons & $\lambda_{Z}$ & $\Delta \mathrm{K}_{Z}$ & $\Delta \mathrm{g}_{1}^{Z}$ & $\Delta \mathrm{K}_{\gamma}$ & $\lambda_{\gamma}$ \\
\hline$W Z,\left(\mathrm{M}_{T}\right)$ & {$[-0.015,0.013]$} & & {$[-0.011,0.034]$} & & \\
$W \gamma\left(p_{T}^{\gamma}\right)$ & & & & & {$[-0.05,0.02]$} \\
$W W,\left(\mathrm{M}_{T}\right)$ & & {$[-0.035,0.073]$} & & {$[-0.088,0.089]$} & \\
\hline$W W$, LEP & & & {$[-0.051,0.034]$} & {$[-0.105,0.069]$} & {$[-0.059,0.026]$} \\
\hline \hline
\end{tabular}

Table 1: $95 \%$ C.L. on charged TGC's from $W W, W Z, W \gamma$ final states with $10.0 \mathrm{fb}^{-1}$ of data [5]. Only the most stringent limits are shown. The variables used in the coupling fit are shown as well some of the existing limits (the most stringent).

\begin{tabular}{|c|cccc|}
\hline \hline Dibosons & $f_{4}^{Z}$ & $f_{5}^{Z}$ & $f_{4}^{\gamma}$ & $f_{5}^{\gamma}$ \\
\hline$Z \rightarrow \ell \ell \ell \ell+$ & & & & \\
$Z \rightarrow \ell \ell \nu v$ & {$[-0.009,0.009]$} & {$[-0.009,0.009]$} & {$[-0.010,0.010]$} & {$[-0.011,0.010]$} \\
\hline LEP & {$[-0.30,0.30]$} & {$[-0.34,0.38]$} & {$[-0.17,0.19]$} & {$[-0.32,0.36]$} \\
\hline \hline
\end{tabular}

Table 2: $95 \%$ C.L. limits on neutral TGCs from $Z Z$ final states for $10.0 \mathrm{fb}^{-1}$ of data. The other anomalous couplings are assumed to be zero. The $95 \%$ C.L. limits from LEP are also shown.

plings (TGC) and therefore the non Abelian structure of the electroweak theory. Within the Standard Model (SM), the trilinear vertices $W W \gamma$ and $W W Z$ occur, while those involving only neutral gauge bosons are absent. If no Higgs is found, diboson production studies are expected to play an important role in understanding the electroweak symmetry breaking mechanism. Finally diboson events represent a background in Higgs and New Physics searches.

All expected diboson processes have been already observed at Tevatron in leptonic channels; at LHC significant improvements are expected since the higher energy allows to explore a more favorable kinematic region and cross-sections are $\sim 10$ times higher. The CMS analyses uses cut-based analyses [13, 14]. In a recent analysis of the $W Z \rightarrow \ell v \ell \ell$ final state [14] data driven methods (based on the use of control samples) are proposed to extract the QCD background. It is estimated that it is possible to reach a $5 \sigma$ significance of the $W Z$ signal with less than $350 \mathrm{pb}^{-1}$ at $95 \%$ C.L..

ATLAS compares cut based analyses with analyses based on the boosted decision tree (BDT) technique [5]. The BDT technique allows an improved sensitivity. With $0.1 \mathrm{fb}^{-1}$ and assuming $20 \%$ systematic uncertainties on the cross section measurements, SM signals of $W W, W Z, W \gamma, Z \gamma$ will be established with a significance better than $5 \sigma\left(\sim 1 \mathrm{fb}^{-1}\right.$ is needed for $\left.Z Z\right)$. Systematics uncertainties (luminosity, lepton selection and identification efficiency, PDF, factorization scale) will dominate the cross-section measurements starting from a luminosity of 5-30 $\mathrm{fb}^{-1}$, depending on the analysis.

The charged TGC are usually taken as $\mathrm{g}_{1}^{Z}, \mathrm{~K}_{V}, \lambda_{V}(\mathrm{~V}=\gamma, Z)$. Within the SM, their values are $\mathrm{g}_{1}^{Z}=$ $\mathrm{K}_{V}=1$ and $\lambda_{V}=0$. It is common then to redefine: $\Delta g_{1}^{Z}=g_{1}^{Z}-1=\Delta K_{V}=K_{V}-1=0$. For ZZ final states, the neutral TGC are usually taken as $\mathrm{f}_{4}^{V}, \mathrm{f}_{5}^{V}(\mathrm{~V}=\gamma, Z)$ and their expected values in SM are $\mathrm{f}_{4}^{V}$, $\mathrm{f}_{5}^{V}=0$. Anomalous values of TGC's lead to increased cross sections especially at high boson $p_{T}$ and di-boson transverse mass, $M_{T}^{V}$. The corresponding spectra are used to set limits on anomalous TGCs at the 95\% C.L.. Tables 1 and 2 show these limits for $10 \mathrm{fb}^{-1}$ of ATLAS data. The prospects 
are that it will be possible to improve up to a factor $\approx 10$ the present limits.

\section{Conclusions}

Even after having found a Higgs signal, precision electroweak measurements will be important since indirect constraints will help the interpretation of its nature and of the underlying theory. The LHC will be a $W, Z$, top factory. The goals of obtaining per experiment $\Delta M_{W}<10 \mathrm{MeV}$, $\Delta M_{t}<1 \mathrm{GeV}, \Delta \sin ^{2} \theta_{\text {eff }} \approx$ few $10^{-4}$ are expected to be within reach. Electroweak dibosons signals are expected to be established after having collected a statistics ranging from $100 \mathrm{pb}^{-1}$ up to $1 \mathrm{fb}^{-1}$. The present limits on anomalous couplings are expected to be improved significantly with $10 \mathrm{fb}^{-1}$. Ultimately, the main concern at LHC will be to understand and control the systematics. This will come from data driven methods, from the use of independent analysis methods and the interplay between improved measurements and theoretical developments.

\section{References}

[1] Peter Renton, 'Global Electroweak Fits and the Higgs Boson Mass', ICHEP 2008 Proceedings.

[2] The ATLAS Collab., G. Aad et al., 'The ATLAS Experiment at the CERN Large Hadron Collider', 2008 JINST 3 S08003.

The CMS Collab., S. Chatrchyan et al., 'The CMS experiment at the CERN LHC', 2008 JINST 3 S08004.

[3] Ashutosh Kotwal, 'Measurements of the $W$ mass', ICHEP 2008 Proceedings.

[4] The CMS Collab., 'Prospects for the precision measurement of the $W$ mass with the CMS detector at the LHC', CMS NOTE 2006/061.

[5] The ATLAS Collab., 'Expected Performance of the ATLAS Experiment, Detector, Trigger and Physics', CERN-OPEN-2008-020, Geneva, 2008, to appear.

[6] N. Besson et al., 'Re-evaluation of the LHC potential for the measurement of $m_{W}$ ', SN-ATLAS-2008-70.

[7] The CMS Collab., 'Top quark mass measurement in single leptonic ttbar events', CMS NOTE 2006/066.

[8] The CMS Collab., 'Measurement of top-pair cross section and top-quark mass in the di-lepton and full-hadronic channels with CMS', CMS NOTE 2006/077.

[9] C. S. Hill, J. R. Incandela and J. M. Lamb, 'Method for measurement of the top quark mass using the mean decay lenght of $b$ hadrons in ttbar events', Phys. Review D 71, 054029 (2005)

[10] The CMS Collab.,'Determination of the top mass with exclusive events $t \rightarrow W b \rightarrow \ell v J / \Psi X^{\prime}, \mathrm{CMS}$ NOTE 2006/058.

[11] M. Beneke et al., 'Top Quark Physics', arXiv:hep-ph/0003033v1, Report of the '1999 CERN Workshop on Standard model physics (and more) at the LHC' (1999) 419-529.

[12] N.Kidonakis and R.Vogt, 'The theoretical top quark cross section at theTevatron and the LHC, arXiv:0805.3844v1,25 May 2008.

[13] The CMS Collab., 'Study of Di-Boson Production with CMS at LHC', CMS NOTE 2006/108.

[14] The CMS Collab., 'Study of the process $p p \rightarrow W Z \rightarrow \ell^{ \pm} v \ell^{+} \ell^{-}(\ell=e, \mu)$ at CMS', CMS PAS EWK-08-003. 